\section{Abstractions}

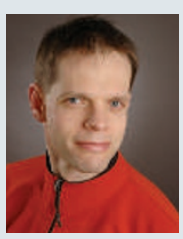

\section{CO-AUTHOR}

As electronic devices get smaller, the magnetic properties of individual atoms will become increasingly important. But the ability to look at an atom's magnetic moment, or spin, at the nanometre scale has, until recently, been limited to conducting surfaces, which can be investigated using polarized scanning tunnelling microscopy. To overcome this barrier, Alexander Schwarz and his colleagues at the University of Hamburg in Germany needed a microscope with atomic resolution that could scan non-conducting materials. They built a magnetic exchange force microscope, based essentially on an atomic-force microscope but with a magnetic tip. Their microscope not only visualizes atoms but also senses their spin. The Hamburg team has used the microscope to probe both the arrangement and spin of surface atoms on the antiferromagnetic insulator nickel oxide (see page 522).

You weren't the only one trying to build this type of microscope. Why has the technology only now matured? Achieving atomic resolution has become routine with atomic-force microscopy, but that detects chemical forces around ten times greater than the magnetic exchange forces. Microscopes as stable and forcesensitive as ours are rare in the scientific world. And we were the first to use a magnetic field to increase exchange forces.

What was the main technical difficulty? We had to get the tip of our microscope very close to the sample surface to image the spin structure of the nickel oxide. If the tip wasn't close enough, the exchange force of spins at the sample surface with the spin of the probe was too small and was masked by atomic forces. If the tip was too close, it hit the sample surface and got damaged.

What's so interesting about atomic spin? Spins and their interactions determine the magnetic properties of a material. Magnetic materials are widely used for data storage and in sensors. As nanotechnology makes components smaller and smaller, there are more interfaces of different materials for a given volume, where spins can interact. The smaller the magnetic structures, the more important is each single spin.

Why should we be interested in nonconducting materials?

It's impossible to build any electronic component without them. Recently developed 'exchange bias systems' in sensors and storage elements use isolating antiferromagnets similar to nickel oxide to pin a ferromagnetic layer against external magnetic fields.

\section{MAKING THE PAPER}

\author{
Barry Dickson
}

\section{A pheromone receptor that controls mating behaviour in Drosophila.}

About 5 years ago, Barry Dickson's group at the Research Institute of Molecular Pathology in Vienna started studying the mating habits of the fruitfly Drosophila. His goal was to unravel the neural circuits in the brain that control behaviour, and mating seemed like a good starting point. "It is a robust behaviour," says Dickson. "It is something flies are really good at doing."

In flies, gender-specific behaviour is controlled by a gene called fruitless, which is expressed in the nervous system. In 2005, Dickson and others found that fruitless is expressed in three out of the 50 types of neurons that sense odors. He thought these 'fruitless' neurons might detect sex pheromones - chemicals produced by animals to let other members of the species know things like the animal's sex or willingness to mate.

Dickson had a clue as to the pheromone involved. One of the three types of fruitless neurons also expressed a receptor thought to sense the male sex pheromone 11-cis-vaccenyl acetate (cVA). Graduate student Amina Kutrovic engineered flies lacking the putative $\mathrm{cVA}$ receptor, and with the help of postdoc Alexandre Widmer examined the flies' behaviour.

Normally, male flies court females and not males, and females mate with males. But mutant male flies lacking the cVA receptor often courted males, and mutant females were less willing to have sex. So it appeared that activating the receptor for cVA had opposing functions: turning males off and females on.

To test this idea further, the team engineered male flies to produce a receptor for a female moth sex pheromone in the same neurons that normally express the cVA receptor. They then rubbed the moth pheromone on female fly abdomens so that the female flies now smelled

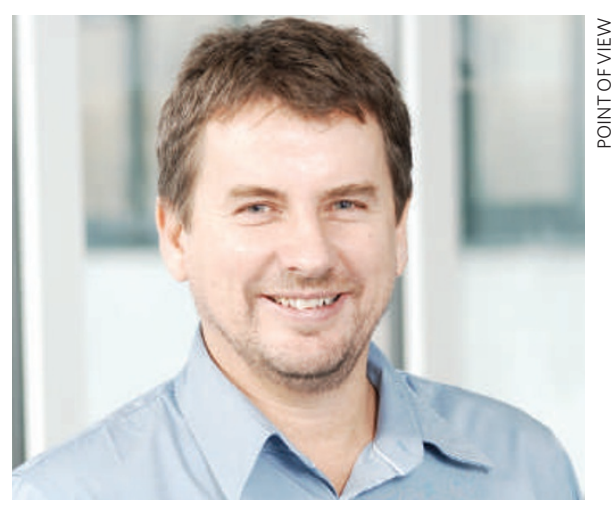

like female moths. Male flies expressing the moth pheromone receptor shunned these females, whereas those without it were happy to court them (see page 542). This seemingly counterintuitive result told the scientists that the activation of neurons normally expressing the cVA receptor is responsible, and sufficient, for suppressing courtship by males.

Everyday odours, such as the smell of rotting fruit, activate many different receptors on different neurons, and the odour identity is encoded by the combination of activated receptors. But odours of particular biological significance, such as pheromones, may activate a single class of olfactory neurons, thus communicating an unambiguous signal to the brain.

Dickson believes that the difference in responses to cVA between males and females depends on how the signal is processed in the brain. "The sensory processing appears to be the same in both sexes," says Dickson. "We don't know exactly where the difference arises, but it does not seem to be in the olfactory neurons themselves."

His group is now tracking down the higherorder pathways that process the signals from the cVA receptor. "We want to know how such chemical signals activate neural circuits to elicit behaviour," he explains. "The fruitless and Or67d genes are not present in humans, and so these findings cannot be directly translated to human behaviour. But we may be able to extend the results to other species by looking for analogy rather than homology."

\title{
KEY COLLABORATORS
}

While trying to build a better mathematical model for recurrent measles epidemics, biomathematics professor Lewi Stone at Tel Aviv University in Israel and his colleagues came up against a major challenge. Sometimes epidemics come every year, sometimes every two years, sometimes every few years; it's hard to predict.

They found this feature

difficult to capture in models even the nonlinear, chaotic models that they'd devised. But the breakthrough came, thanks in part to the fresh perspective of then-graduate student Ronen Olinky. "He decided to look at the problem from a completely different angle," says Stone.

Instead of trying to make a model that generates an epidemic each year, Stone and co-author Amit Huppert, on Olinky's suggestion, decided to investigate why in some years the epidemic doesn't come.

They looked at one major influencing factor: a population's susceptibility how many susceptible individuals (those who have never had measles nor been vaccinated) remain after an outbreak (see page 533). Seasonality is also key outbreaks occurring later in the disease 'season' affect fewer individuals and leave more susceptible ones behind. Their model is geared to measles, although Stone is now trying to tweak it for seasonal influenza. 